\title{
Modelling Insight to Ball Eyes for Higher Dimensional Hyperspace Vision
}

\section{Shaikh $S^{*}$ \\ Aditya Institute of Management Studies and Research (AIMSR), India}

*Corresponding author: Sadique Shaikh, Aditya Institute of Management Studies and Research (IMSR), Jalgaon, India, Email: sids_nsk@rediffmail.com

\section{Letter to Editor}

Volume 5 Issue 2

Received Date: July 16, 2021

Published Date: July 26, 2021

DOI: $10.23880 /$ psbj-16000183

\section{Letter to Editor}

To understand this complicated conceptual idea let me begin first with the definition of VISION and then after DIMENSIONS (Figure 1). The Vision is ability to acquire surrounding with input light, shapes, places, color to brain to create animated CONSCIOUSNESS in the help of Brain call Observable Life, Planet, Universe and Multiverse. Equally Vision also important to grow Brain Intelligence and Control to enhance, develop and shape planet earth and at present observable Universe. Now I would like to define term Dimensions as the ability of Eyes to scan surrounding available Vision with Left, Right, Top, Bottom, Reflection, Rotation, Transformation, Spinning and Diagonal with all possible angles and geometry and provide data to Brain to create high definition Consciousness of environment, planet, universe and multiverse. For our understanding purpose we labeled the Dimensions which we (Human) can see and understand are 3D Three-Dimensional World as X-Axis, Y-Axis and Z-Axis with additional fourth Dimension virtually TIME and Brain create 3D consciousness using X, Y and Z Axis's Vision Data after input processed. Since Human Eyes has ability to see in three dimensions hence very easily can see Dot [Zero Dimension/No Axis] like confine and particles, Line [One Dimension/X-Axis] like Rays, Linear object shaped to line shape, Plane [Two Dimensions / X,Y Axis's] like paper, mat, kite or any Plane object shaped plane shape and finally Cube/Box [Three Dimensional/X,Y and Z Axis's] here we can see our complete world and observable Universe, BUT only with our sense our understanding which is our limitation and ability to see what our eyes provide to our brain and our brain generate meaning of respective input as "3D-Consiousness Out" for LIFE and scenario is different lot of might be we every second missing to vision from our surrounding, world to universe because we can see and understand only "Three Dimension". My meaning to say we are not species with High
Quality vision even some animals, reptiles, birds and insect has good vision as compare to human eyes. To understand "Dimensional-Vision" some depicts as given below.

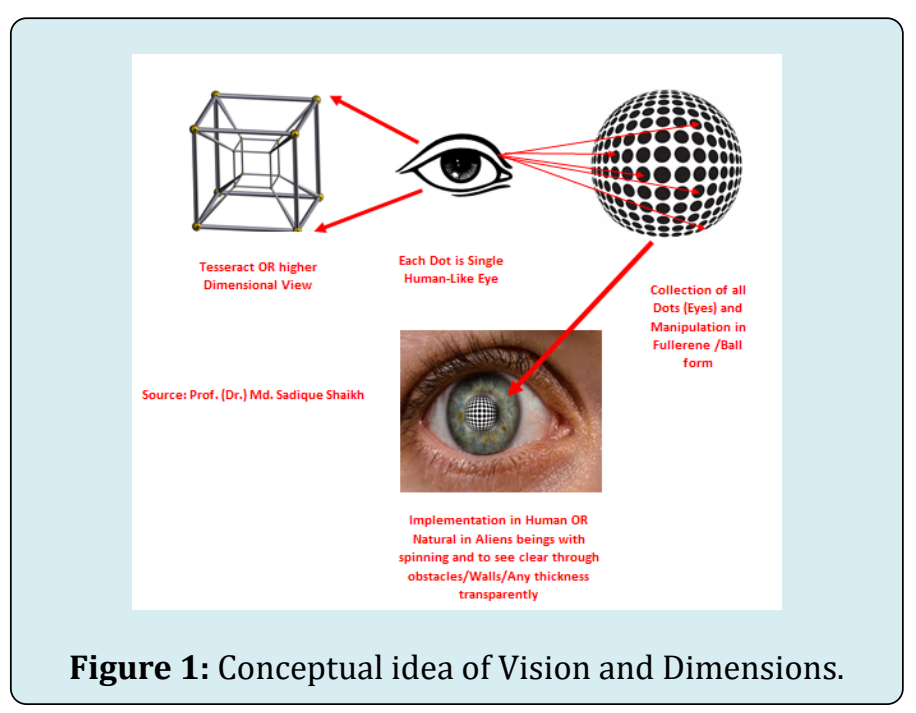

Figure 2A shows Three Dimensional (Cube) Species Visiting Two Dimensional (Plane) Species. This figure gives you fusion of 2D (X,Y Axis's) and 3D (X,Y,Z Axis's) Species at interface or point of "Dimensions Interchange/Transform" with switching at interface from 2D to 3D and reverse if you observe it deeply. Some possibilities in 2D species as we rotate from left to right or right to left position of body elements/ organs like heart also get changed which impossible in 3D species display in Figure 2B. Figure 2C is comparison of 2D and 3D for same species with elements/objects and Figure 2D is how species switching from Two Dimensional world to Three Dimensional world. If human got ability to enter in 2D world they will act like Super-power human with God- 
like abilities for example "Appear and Disappear" from Top (Z-Axis) in 2D Plane because 2D world don't have additional Z-Axis what 3D world has.

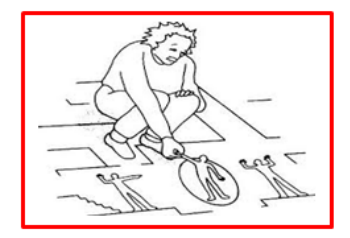

A

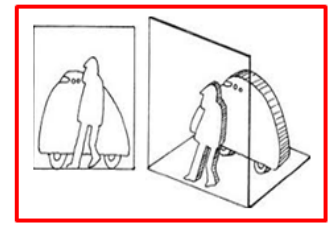

C

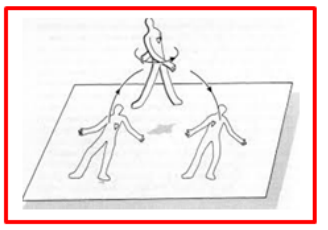

B

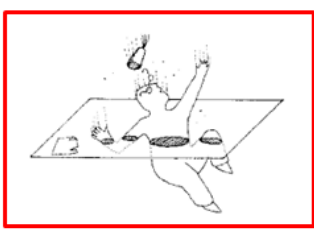

D

Figures 2: A) Three Dimensional (Cube) Species Visiting Two Dimensional (Plane) Species; B) 3D species display; C) Comparison of 2D and 3D for same species with elements/ objects; D) how species switching from Two Dimensional world to Three Dimensional world.

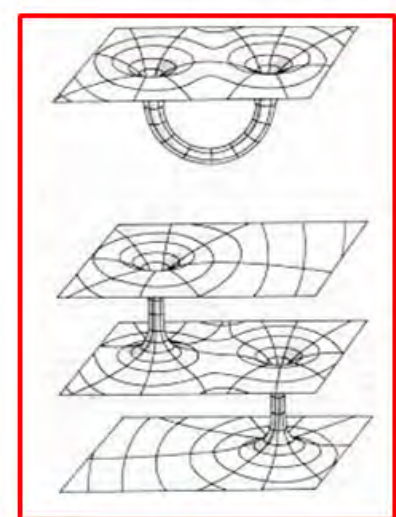

$\mathbf{E}$

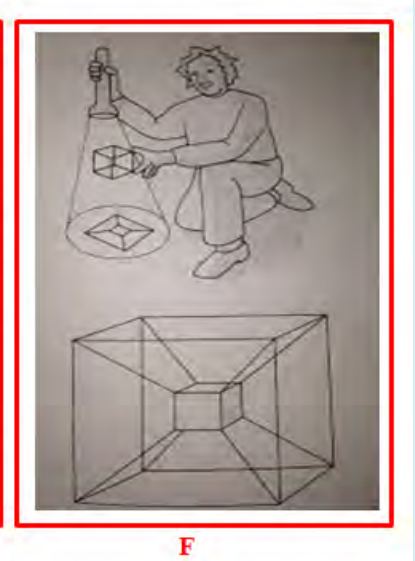

Figures 2: E) Thick interface, hyperspace, wormholes; F) The magical geometry of Tesseract in 2D projection, 3D vision Physical and 4D simulation.

Hence now I am coming to my discovery "Ball Eyes" and how it will helpful in near future to vision and explore higher dimensions of Space and Hyperspace. If our brain gives ability to our eyes or by any Biological (Natural) or External man-made (Artificial) means our eyes got ability of "Backward Vision-Switching" from 0, 1, 2, 3 dimensions world and back our vision would like this as Human vision switch from 3D to 2D world all appearance in their vision just mixed colors complicated intersect planes of various "Length and Width" with absence of "Height". Similarly if switching from 2D to $1 \mathrm{D}$ complete vision appearance would be different "Length" mixed colors and different lengths complicated vibrational lines with absence of "Width and Height". And finally as switching from 1D to 0D all "Length, Width and Height" absent and vision appearance would only colorful vibrational dust/ vibrational particles seems to be vanishing and 'every entity just dust'. Means with backward vision brain consciousness convert species to Cubes to planes, plane to fine lines and finally in diminishing particles (Dots) dust means "3D world Human has No Existence or Death-like" in Zero-dimension world and vision and unable to do anything. But in 3D world, Vision and Consciousness Human Species capable to do lot of things and doing extra ordinary things with help of Quantum Physics, Science and Technology, can say its "Stable" or "Comfortable" Vision and consciousness of 3D world to see, where we also use additional dimension as "Space" and "Time" to locate and relocate our visions like When to meet (Time) and where to meet (Space). My claim is as we and our vision became weak to weaker as switch backward from 3D vision to 2D, 1D and OD (No existence). Similarly if we got ability in near future of "Forward VisionSwitching" from 3D to 4D, 5D,6D,7D....... co called "Higher Dimensions of Space" we will became strong to stronger and strongest with our vision and consciousness having "Alienslike" super powers like "Appear and disappear", "Travel with speed of light", "teleport from one space/universe to another space/universe using thoughts power", "able to travel back and forth in Time-Line and switch easily to our past, present and future", "Eyes have many dimensional Tesseract like vision to directly pass from thick interface, hyperspace, wormholes etc." and many more as display in Figure E Wormholes interconnect three different "Spaces" in group called "Multiverse or Hyperspace" and Figure F display the magical geometry of Tesseract in 2D projection, 3D vision Physical and 4D simulation. Therefore to capture such an extreme extraordinary vision we need extreme eyes "Might be some Aliens species have in Universe/Multiverse" with lots of thousand "Retina's (like human eyes) engineer, design and manipulate in "Fullerene or Ball-like" manner I named the term "Ball Eyes" with all direction "Spinning" ability either naturally in some Aliens with such a Ball Eyes on their bodies part (Not compulsory only in head like human species) and can be implement in human Artificially but with ability of "Ball Eye Spinning" and "Ball Eyes can see outside from inside part of it in body transparently" where thousand Dots represent to thousand eyes stated in Ball/Fullerene for "Ball-Eyes" with higher dimensional hyperspace vision ability to make mankind Ultra-Immortal as shown in my traced Model at the begging. 